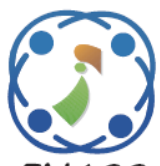

\title{
Multipath Data Transmission in IoT Networks Using Fractional Firefly Algorithm and Chicken Swarm Optimization
}

\author{
Salem Abdulla Awadh Ba Hmaid ${ }^{1 *}$ \\ Vasanthi Varadharajan ${ }^{2}$ \\ ${ }^{I}$ Computer Science Department, Rathinam College of Arts and Science, \\ Bharathiar University, Coimbatore, India \\ ${ }^{2}$ Department of Information Technology, \\ Sri Krishna Adithya College of Arts and Science, Bharathiar University, Coimbatore, India \\ * Corresponding author's Email: salem100323@gmail.com
}

\begin{abstract}
Integration of Wireless Sensor Networks (WSN) with the Internet Protocol (IP) has led to the development of Internet-of-Things (IoT) networks with high connectivity and improved data transmission with limited power supplies. Hence, it is necessary to utilize a high performance multi-path routing protocol to avoid energy constraint problems and network connectivity issues. This paper presents an optimal multi-path routing protocol using multi objective algorithms namely Fractional Firefly algorithm with Chicken Swarm Optimization $($ FFA+CSO) to resolve the energy constraint problem. First the network is clustered and a Cluster Head $(\mathrm{CH})$ is selected to initiate inter-cluster and intra-cluster communication. Fractional Firefly algorithm (FFA) has been developed for this purpose by overcoming the slow convergence and local optima problem of firefly algorithm. FFA selects $\mathrm{CH}$ based on energy, delay, link quality and lifetime. Then the routes are formed and optimal route is selected using Chicken Swarm Optimization (CSO) based on energy, inter and intra-cluster delay, link quality, lifetime and hop count. The proposed FFA+CSO routing protocol is evaluated using Network Simulator-2. Results obtained for 100 nodes showed that FFA+CSO provided efficient multipath data transmission for WSN based IoT networks with $24 \%$ less delay, $28 \%$ greater throughput, $18.7 \%$ lesser energy consumption, $21.6 \%$ longer lifetime, $20 \%$ higher PSNR and $37.5 \%$ less number of hops than the existing routing models.
\end{abstract}

Keywords: Wireless sensor networks, Internet-of-things, Energy constraint problem, Multipath routing, Cluster head, Firefly algorithm, Fractional firefly algorithm, Chicken swarm optimization.

\section{Introduction}

Internet-of-Things (IoT) is an innovative paradigm that has gained immense attention due to its efficient and novel pattern of turning objects into smart devices to enable connection to internet [1]. Recently, the research interest in IoT has been rapidly increasing in the fields of academia; healthcare, smart cities, environmental monitoring and industrial automation that utilize IoT primarily for efficient data gathering and transmission [2]. In recent years, IoT applications have utilized the concepts of wireless sensor networks (WSN) which has garnered significant attraction for research applications [3]. In combination with the radio frequency identification (RFID) technology, the IoT employs the WSN technology to support applications in various domains such as smart systems, smart cities, smart healthcare systems, environmental monitoring, traffic monitoring, military applications, etc. WSN based IoT ensures better connectivity among the connected things and enable them to provide effective transmission. This collaboration allows the WSN based IoT to utilize the WSN routing protocols for data transmission but the battery constrained nature of the network limits the power utilization and reduce the overall lifetime.

Energy efficiency is one of the serious concerns in the mass organization of WSN based IoT applications. In IoT, sensor nodes are restricted by limited batteries and transmission range. Hence the 
WSN techniques must be ensured to provide energy efficiency and only then the WSN based IoT can be economically feasible for its enormous applications [4]. The routing protocol must also assure the characteristics of WSN, such as dynamic topology, limited power, memory capacity, and high transmission for IoT applications [5]. The existing routing protocols do not consider all the energy components and hence does not provide complete energy efficiency to IoT sensors. Hence energyefficiency and the quality-of-service (QoS) parameters are prominent in routing protocols [6]. Most protocols are built upon either Adhoc OnDemand Distance Vector (AODV) or Dynamic Source Routing (DSR) models [7]. In WSN based IoT networks, AODV models are preferred for the objective of minimizing the broadcast with better tackling of the issue of loops in routing in WSN based IoT networks. The majority of routing algorithms prefer the selection of optimal paths for providing efficient routing with minimizing the total energy consumption [8]. However, the optimal path concept divides the network and creates the energyhole problem due to continuous usage of single optimal path and also the non-uniform energy drain rate in the network nodes. These problems contradict the efficiency of minimized total energy consumption and fail the routing protocol to achieve the intended results.

This paper emphases on resolving these problems and develop an energy efficient multipath routing protocol using $\mathrm{FFA}+\mathrm{CSO}$ based on multiple objectives. The important considerations are load balancing, reduced and uniform energy consumption, minimized packet retransmission and less packet drop rate. To realize these goals, the proposed FFA+CSO based routing model considers the parameters such as energy, delay, link quality and lifetime for $\mathrm{CH}$ selection while energy, inter and intra-cluster delay, link quality, lifetime and hop count for optimal path selection. These improvements enable the network to prolong its lifetime with effective solution for energy-hole problem and non-uniform energy drain rate problems. The rest of the paper is ordered as: section 2 presents the discussion on related works. Section 2 introduces and explains the proposed FFA+CSO based routing model. Section 4 presents the simulation and evaluation results and section 5 presents a conclusion of this paper.

\section{Related works}

Many recent works have aimed to address the energy constraint problem for improving the data transmission in real-time WSN based IoT applications. Machado et al, [9] developed a WSN routing protocol based on energy and link quality for IoT applications. However, it is much suitable for the small scale applications only due to proportional performance on node density. Shen et al, [10] presented energy efficient centroid-based routing protocol for WSN-assisted IoT which reduces the energy drain rate by selecting $\mathrm{CH}$ on rotational basis. This model supports only single-hop transmission while multi-hop transmission are not effective as the base station is mostly situated at the network center. Nguyen et al, [11] introduced energy-harvestingaware routing algorithm for WSN-based IoT applications using energy back-off parameter. This approach has also been extended by Nguyen et al, [12] to develop distributed energy-harvesting-aware routing algorithm. Both these routing algorithms have provided better data transmission in IoT, but the models do not reduce the packet delay and subsequent packet drops.

$\mathrm{CH}$ selection plays a prominent role in energy consumption and also deciding the routing performance. Singh and Lobiyal, [13] proposed the use of particle swarm optimization (PSO) for $\mathrm{CH}$ selection based on the residual energy, minimum average distance and number of head nodes. Rana and Zaveri, [14] proposed Synthesized CH selection and routing using Genetic algorithm (GA) to improve network lifetime. Rao et al, [15] also employed PSO for $\mathrm{CH}$ selection based on intracluster distance, sink distance and residual energy of sensor nodes. Kumar et al, [16] developed FABC+ Exponential Ant Colony Optimization (EACO) algorithms for energy efficient routing. $\mathrm{CH}$ selection is done using FABC based on energy, delay and location while routes are optimized using EACO based on energy, distance, intra cluster delay and inter cluster delay. Similarly, Dhumane and Prasad, [17] proposed Fractional Gravitational Grey Wolf Optimization (FGGWO) based energy aware routing in which the Fractional Gravitational Search Algorithm (FGSA) selects the $\mathrm{CH}$ and FGGWO selects optimal path based on energy, inter and intracluster distance, delay and lifetime. These approaches reduce the cost of locating optimal $\mathrm{CH}$ but the energy balancing properties are not satisfied during transmission.

From the literature, it can be inferred that the optimal route selection models have performed significantly better than other models. It can also be seen that these models too have their own limitations in terms of energy-hole problem. To overcome these limitations, the proposed model 
Table 1. Notations and definitions

\begin{tabular}{|c|c|}
\hline Notation & Definition \\
\hline$P_{i}$ & Initial node power \\
\hline$P_{T}$ & $\begin{array}{l}\text { Power consumed for } \\
\text { transmission }\end{array}$ \\
\hline$P_{R}$ & $\begin{array}{lll}\text { Power } & \text { consumption } & \text { for } \\
\text { reception } & & \\
\end{array}$ \\
\hline$i, j$ & Sensor nodes \\
\hline$D_{t}$ & Distance between nodes $\mathrm{i}$ and $\mathrm{j}$ \\
\hline$P_{\text {cons }}$ & Constant power \\
\hline$P_{a m p}$ & Power amplification \\
\hline$D_{0}$ & Threshold distance \\
\hline$n_{1}, n_{2}$ & IoT nodes redefined \\
\hline$t$ & Time \\
\hline $\begin{array}{c}\left(u_{1}, v_{1}\right) \text { and } \\
\left(u_{2}, v_{2}\right)\end{array}$ & Current location of nodes \\
\hline $\begin{array}{c}\left(u_{1}^{\prime}, v_{1}^{\prime}\right) \text { and } \\
\left(u_{2}^{\prime}, v_{2}^{\prime}\right)\end{array}$ & Updated new locations of nodes \\
\hline$T_{r i}$ & Receive time of i-th packet \\
\hline$T_{s i}$ & Sending time of i-th packet \\
\hline$n$ & Total number of data packets \\
\hline$d$ & $\begin{array}{l}\text { Distance between source and } \\
\text { destination nodes }\end{array}$ \\
\hline$\varepsilon_{0}$ & $\begin{array}{l}\text { total non-rechargeable initial } \\
\text { energy }\end{array}$ \\
\hline$\lambda$ & average broadcasting rate \\
\hline $\mathbb{E}\left[E_{w}\right]$ & expected unused energy \\
\hline $\mathbb{E}\left[E_{r}\right]$ & expected reporting energy \\
\hline$L_{q}$ & Link quality \\
\hline$A G_{r}$ and $A G_{t}$ & $\begin{array}{l}\text { antenna gain of receiver and } \\
\text { transmitter }\end{array}$ \\
\hline$n_{x}^{y}$ & fraction of $\mathrm{n}$ at location $(x, y)$ \\
\hline$l$ & Dimension of fractional input \\
\hline$c$ & Coefficient of fractional input \\
\hline$\gamma$ & Absorption coefficient \\
\hline$F \beta$ & Fractional attractiveness \\
\hline$\beta_{0}$ & Initial attractiveness \\
\hline$F T^{\alpha}$ & Fractional time period \\
\hline$F \alpha$ & Fractional number of iterations \\
\hline$m$ & Number of iterations \\
\hline$D^{\alpha}(r)$ & $\begin{array}{l}\text { minimum distance of fireflies } \\
\text { with required intensity }\end{array}$ \\
\hline$\alpha$ & $\begin{array}{l}\text { non-integer parameter for step- } \\
\text { size control }\end{array}$ \\
\hline$S^{\alpha}$ & increasing intensity index \\
\hline$\alpha_{F}$ & $\begin{array}{l}\text { fractional parameter to control } \\
\text { the step size }\end{array}$ \\
\hline$\epsilon$ & Gaussian distribution vector \\
\hline$F_{p}$ & $\begin{array}{l}\begin{array}{l}\text { fitness function for path } \\
\text { selection }\end{array} \\
\end{array}$ \\
\hline$W$ & Path transmission range \\
\hline$\rho$ & Density of node deployment \\
\hline $\operatorname{rand}\left(0, \sigma^{2}\right)$ & $\begin{array}{l}\text { Gaussian distribution with mean } \\
0 \text { and standard deviation } \sigma^{2}\end{array}$ \\
\hline$X_{i, j}^{T}$ & location of current best chicken \\
\hline$X_{i, j}^{T+1}$ & location of next best chicken \\
\hline$S_{1}, S_{2}$ & coefficient of social factors in \\
\hline
\end{tabular}

\begin{tabular}{|c|l|}
\hline & search space \\
\hline$r 1, r 2$ & Index of rooster and chicken \\
\hline$f_{i}, f_{r 1}$ and $f_{r 2}$ & $\begin{array}{l}\text { fitness of } \mathrm{i} \text {-th hen, rooster with } \\
\text { index } r 1 \text { and rooster/hen with } \\
\text { index } r 2\end{array}$ \\
\hline$a b s\left(f_{i}\right)$ & absolute value of fitness \\
\hline$\varepsilon$ & $\begin{array}{l}\text { smallest constant to avoid zero- } \\
\text { division error }\end{array}$ \\
\hline$X_{m, j}^{T}$ & $\begin{array}{l}\text { location of the j-th chick's } \\
\text { mother }\end{array}$ \\
\hline$f l$ & Food forage parameter of chick \\
\hline $\mathrm{X}(\mathrm{i}, \mathrm{j})$ & Data objects \\
\hline
\end{tabular}

introduces $\mathrm{FFA}+\mathrm{CSO}$ algorithms in the routing protocol.

\section{Methodology}

Table 1 presents the list of notations used in this paper and their definitions.

\subsection{System model}

WSN is composed of N sensor nodes, which can sense and obtain data. Each node in the given network is capable of shifting their transmission energy and also has the capacity to act as both $\mathrm{CH}$ as well as the member nodes. The sensor nodes are clustered into groups and each of the group is headed by a $\mathrm{CH}$ selected based on certain objectives [17]. Each IoT node collects the data and transmits them to the $\mathrm{BS}$ through the $\mathrm{CH}$ but it consumes considerable amount of energy for these processes. Hence the $\mathrm{CH}$ is selected on energy and distance. Assign a simulation area with dimension, BS, and $\mathrm{k}$ IoT nodes distributed as clusters each headed by a $\mathrm{CH}$. The proposed scheme could be employed in any WSN based IoT routing protocols to implement cooperation among nodes.

\subsubsection{Energy model}

Considering each sensor has an initial energy $P_{i}$ and if the key factors of energy consumption is based on packet reply and broadcast. $P_{T}$ is the power utilization of node $\mathrm{i}$ to transmit a data packet to its nearby node $\mathrm{j}$ and $P_{R}$ is the power utilization of node i while receiving a data packet from its nearby node j. If the sink node reflects unlimited energy and maintains movement until the end of the network lifetime, which is denoted as the time until the first node expires due to energy depletion. The objective of optimization problem is the selection of optimal routing strategy and the optimal $\mathrm{CH}$ selection at each sink location thus the network lifetime is maximized for a given order of node locations. It 
provides better network lifetime by using linear programming model. Power consumption for transmitting $\mathrm{L}$ bits of data at distance $D_{t}$ is given in Eq. (1) and power consumption for receiving $L$ bits of transmitted data packets is given in Eq. (2).

$$
\begin{gathered}
P_{T}=\left\{\begin{array}{cc}
P_{\text {cons }} \times L \times P_{a m p} \times D_{t}^{2} & \text { if } D_{t}<D_{0} \\
P_{\text {cons }} \times L \times P_{a m p} \times D_{t}^{4} & \text { if } D_{t} \geq D_{0}
\end{array}\right. \\
P_{R}=P_{\text {cons }} \times L
\end{gathered}
$$

Where $P_{\text {cons }}$ is the constant power, $P_{T}$ power consumption for transmission of packets, $\mathrm{L}$ is bits of data, $P_{a m p}$ is power amplification, $D_{t}$ is distance among nodes, $D_{0}$ is threshold distance, $P_{R}$ is power consumption while receiving the packets. The power consumption is reduced significantly in this model during the data transmission and shortest routing path can also be ensured effectively.

\subsubsection{Mobility model}

The mobility model [18] represents the measure of the WSN based IoT nodes in the system based on the location, speed and hop. It discovers the implementation of the scheme in the network for packet data communication. Consider $n_{1}$ and $n_{2}$ be two IoT nodes located at $\left(u_{1}, v_{1}\right)$ and $\left(u_{2}, v_{2}\right)$, respectively. At a time $\mathrm{t}=1$, both the nodes move to a new position $\left(u_{1}^{\prime}, v_{1}^{\prime}\right)$ and $\left(u_{2}^{\prime}, v_{2}^{\prime}\right)$ such that the link of the nodes is within a particular region. The Euclidean distance among these nodes is given as

$$
d(0)=\left|u_{1}-u_{2}\right|^{2}+\left|v_{1}-v_{2}\right|^{2}
$$

The distance among the WSN based IoT nodes at any time 1 in the new positions is calculated as follows,

$$
d(l)=\left|u_{1}{ }^{\prime}-u_{2}{ }^{\prime}\right|^{2}+\left|v_{1}{ }^{\prime}-v_{2}{ }^{\prime}\right|^{2}
$$

Here $\left(u_{1}{ }^{\prime}, v_{1}{ }^{\prime}\right)$ and $\left(u_{2}{ }^{\prime}, v_{2}{ }^{\prime}\right)$ are the new locations obtained via the nodes $n_{1}$ and $n_{2}$ respectively.

\subsubsection{Routing model}

The routing is performed by the protocol built upon the AODV routing algorithm to reduce the amount of retransmissions through generating routes on-demand with high effectiveness. This protocol forms the routing table when source node needs to broadcast data [19]. The loop-free, single path, distance vector protocol based on hop-by-hop routing approach of AODV is utilized and the two main strategies employed are: Route discovery and
Route maintenance. The network is grouped into clusters of similar nodes and a $\mathrm{CH}$ is selected in each cluster using newly developed FFA. Based on the $\mathrm{CH}$, the inter-cluster routes are formed and the routing paths are selected optimally using CSO.

\subsection{Cluster head selection}

The objective parameters namely energy, delay, lifetime, and link quality are considered for selecting the optimal $\mathrm{CH}$ in the WSN based IoT network. The network is categorized into regions of similar nodes called cluster and each cluster contains a set of characteristically similar sensor nodes. The network lifetime can be improved in better way with optimal selection of $\mathrm{CH}$ which reduces the non-uniform energy drain rate. The primary objective is to avert the excess energy consumption and avoid the packet loss owing to various delays. This two metrics will significantly contribute to the increase in network lifetime. The formula for calculating delay, energy, lifetime, and link quality are given below:

$$
\operatorname{delay}\left(T_{d}\right)=\frac{\sum_{i=1}^{n}\left(T_{r i}-T_{s i}\right)}{n}
$$

Here $T_{r i}$ is the receive time of $\mathrm{i}$-th packet, $T_{s i}$ is the sending time of $i$-th packet and $n$ is the total number of packets.

$$
\operatorname{Energy}(e)=(2 \times i-1)\left(P_{t}+P_{r}\right) d
$$

Here $\mathrm{i}$ is the data packet, $P_{r}$ denotes the reception power and $P_{t}$ denotes the transmitter power of the packet $\mathrm{i}$ and $\mathrm{d}$ is the distance from the source to the destination node.

Lifetime is the time a network operates until the first sensor node or the group of nodes in the network runs out of energy. It can be simply defined as the overall network lifetime that is determined by the remaining energy in the network.

$$
\text { Lifetime } \mathbb{E}[L]=\frac{\varepsilon_{0}-\mathbb{E}\left[E_{w}\right]}{P+\lambda \mathbb{E}\left[E_{r}\right]}
$$

Where $\mathrm{P}$ is the constant continuous power depletion of the whole network, $\varepsilon_{0}$ is the total nonrechargeable initial energy, $\lambda$ is the average broadcasting rate, $\mathbb{E}\left[E_{w}\right]$ is the anticipated misused energy or unused energy when the network dies and $\mathbb{E}\left[E_{r}\right]$ is the expected reporting energy consumed by all sensors.

Link quality $\left(L_{q}\right)$ can be estimated based on link expiration time or link energy drain rate based on 
the reception power as $L_{q}$ is directly proportional to reception power.

$$
\begin{aligned}
& L_{q} \propto P_{r} \\
& P_{r}=P_{t} \times A G_{r} \times A G_{t} \times \frac{\lambda^{2}}{(4 \pi d)^{2}}
\end{aligned}
$$

Where $A G_{r}$ and $A G_{t}$ are the antenna gain of receiver and transmitter respectively, and $\lambda$ is the average broadcasting rate. These four parameter values are used to form the fitness function or objective function for $\mathrm{CH}$ selection and formulated fitness function has to be resolved to obtain pareto-optimal solution.

The fitness function $F$ is formulated as the combination of weighted functions of all these four objective parameters.

$$
\begin{gathered}
F=w\left(T_{d}\right) \times f\left(T_{d}\right)+w(e) \times f(e)+ \\
w(\mathbb{E}[L]) \times f(\mathbb{E}[L])+w\left(L_{q}\right) \times f\left(L_{q}\right)
\end{gathered}
$$

Where $f(e)$ is the energy function, $f\left(T_{d}\right)$ is the delay function, $f(\mathbb{E}[L])$ is the lifetime function and $f\left(L_{q}\right)$ is the link quality function and $w\left(T_{d}\right), w(e)$, $w(\mathbb{E}[L]), w\left(L_{q}\right)$ are the weight functions of delay, energy, lifetime and link quality parameters, respectively. The energy parameter is given high priority in this model to ensure energy efficiency and hence the higher weights are assigned to $w(e)$ when the optimal $\mathrm{CH}$ is selected. The $\mathrm{CH}$ is selected using FFA which is an improved version of FA using the fractional theory concept.

\subsubsection{CH selection using FFA}

FFA selects the optimal $\mathrm{CH}$ node for each cluster in the given network. Though efficient, the FA algorithm is not effective for larger networks and hence fails to expand the network lifetime in such cases. This is predominantly due to the exploration and exploitation property of the traditional FA [20]. Hence the theory of fractional calculus [21] is applied to form fractional FA which works better in larger networks and can be applied as fractional groups in each of the regions of the network to improve the search ability. Fractional calculus assumes the use of pseudo-differential operators with real powers of the differential operator. FFA algorithm selects the best node as $\mathrm{CH}$ node based on energy, delay, lifetime, and link quality parameters. FFA improves the memory and hereditary properties of the processes by generalizing the derivative or integral of a function to non- integer orders. This process increases the convergence rate and avoids sinking into local optimum cycle.

First the population of fractional fireflies is initialized as $F x_{i},(i=1,2, \ldots n)$. The fractional theory is applied to the general FA. The fractional formula [21] used for this modification is given by

$$
n_{x}^{y}(l+1)=\gamma n_{x}^{y}(l)+\frac{1}{2} \gamma n_{x}^{y}(l-1)+c^{i}(l+1)
$$

Where $n_{x}^{y}$ is the fraction of $\mathrm{n}$ at location $(x, y), l$ is the dimension of the fractional input and $c$ is the coefficient of fractional input with absorption coefficient $\gamma$. The cost estimation must be performed to determine the feasibility of using fractional FA. The cost function is formulated as

$$
\begin{aligned}
\cos t=F \beta & \times f_{1}+(1-F \beta) \times f_{2}+\left(1-F \beta^{2}\right) \\
& \times f_{3}+\cdots+\left(1-F \beta^{n}\right) \times f_{n}
\end{aligned}
$$

Here $F \beta, F \beta^{2}, \ldots, F \beta^{n}$ is the fractional attractiveness of the $\mathrm{n}$ fireflies and $f_{1}, f_{2}, \ldots, f_{n}$ is the individual fitness of $\mathrm{n}$ fireflies. The cost of FFA is significantly lesser than the FA and hence it is recommended to utilize the FFA in place of FA. The fitness can be computed using Eq. (10) while the Fractional attractiveness $(F \beta)$ can be computed as

$$
F \beta=\frac{1}{F T^{\alpha}} \beta_{0} \exp \left(-\gamma r^{m+F \alpha}\right)
$$

Here $F T^{\alpha}$ is fractional time period and $F \alpha$ is the fractional number of iterations at $\mathrm{m}$ iterations. Then the fractional light intensity (FI) is formulated as

$$
F I=D^{\alpha}(r) \cdot I_{0} \exp \left(-S^{\alpha} \gamma r^{2}\right)
$$

Here $D^{\alpha}(r)$ is a minimum distance of fireflies with required intensity, $\alpha$ is the non-integer parameter for step-size control and $S^{\alpha}$ is the increasing intensity index. The distance and other metrics are computed as in FA. The fractional fireflies are updated using

$$
F x_{i}=\alpha_{F} x_{i}+\frac{1}{2} \alpha_{F} \beta_{0} e^{-\gamma r_{i, j}^{2}}\left(x_{j}-x_{i}\right)+\alpha \epsilon
$$

Here $\epsilon$ is the Gaussian distribution vector, $\alpha$ is the non-integer parameter for step-size control, $\alpha_{F}$ is the fractional parameter to control the step size of the fireflies.

Once the FFA completes its search process, the best node in the cluster is identified which has best fitness function. This node will be selected as the 
$\mathrm{CH}$ and its fitness equation will return the following form

$$
\begin{gathered}
F_{i}=w(e) \times f_{i}^{\text {low } e}+w\left(T_{d}\right) \times f_{i}^{\text {less } T_{d}} \\
+\left(L_{q}\right) \times f_{i}^{\text {high } L_{q}}+w(\mathbb{E}[L]) \times f_{i}^{\text {high } \mathbb{E}[L]}
\end{gathered}
$$

There is diversity amid the members in every population of live creatures in terms of quality and fitness. Global solution of firefly algorithm is aimed to increase the performance of the agents (network) in determining more appropriate solutions by modifying them, develops the quality of firefly's society, thereby the probability of finding the multiple optimal solution can be increased. Algorithm 1 shows the $\mathrm{CH}$ selection process using the above developed FFA.

\section{Algorithm 1. FFA based CH selection}

Generate $\mathrm{n}$ nodes to cover $\mathrm{k}$ randomly initialized fractional fireflies

Select the closest nodes $i$ and $j$ as primary fractional fireflies for comparison

Map the randomly assigned locations along with closest co-ordinates

Begin

While ( $\mathrm{m}<$ Max generation)

If (any fractional firefly (node) is with less lifetime)

Keep the firefly in new location stochastically

Update the solution set

End if

Select the mapped location of $i, j$ along with closest co-ordinates

Estimate the cost function using Eq. (12)

For $\mathrm{i}=1$ to $\mathrm{n}$ (all $\mathrm{n}$ fireflies)

For $\mathrm{j}=1$ to $\mathrm{n}$ (all $\mathrm{n}$ fireflies)

Compute fractional theory based generation (assume $I_{j}$ and $I_{i+1}$ are same)

Compute the energy, delay, link quality and network lifetime parameters

Compute fitness using Eq. (10)

If $\left(F_{j}>F_{i}\right)$

Move i towards j;

End if;

Sort the fractional fireflies

Update the node's locations (fractional firefly update) using Eq. (15)

Restrict the frequent change in the node locations using attractiveness $F \beta$

Update the solutions list

End for $j$

End for $\mathrm{i}$

Rank the fireflies and determine the current best

Return $\mathrm{CH}$

End
The position of the fireflies (nodes) is updated using FA along with the fractional concept. This can resolve the issues in the search process that occur in FA, offering improved location update of nodes. The fractional algorithm is used for the rapid evaluation of the centroid $\mathrm{CH}$ node selection. The fractional algorithm, evaluates the cluster centroid point in the given network. The centroid points initialized are subjected to the intensity and brightness. Once the new points are produced, the fractional calculus based solution point is produced based on the constraint of the random solution points. Therefore several solution points are produced and using the computed solution point, the fitness estimation is performed. After the fitness computation, the centroid point along with the optimal fitness is assumed as the $\mathrm{CH}$ and the procedure of FFA is iterated until the best $\mathrm{CH}$ is ensured. FFA metaheuristic is chosen for its capability of providing optimal solutions for multiobjective problems in larger networks with highly accurate exploration property to avoid stagnation in local optimum position. $\mathrm{CH}$ node selection is performed by using FFA algorithm based on the lower energy consumption, less distance (estimated using delay metric) and higher packet delivery and lower number of hop count nodes. Thus selected $\mathrm{CH}$ performs the functionalities of the leader until it runs out of energy or a better node in the cluster takes its position.

\subsection{Routing path selection using CSO}

The routing path is selected using the CSO optimization algorithm [22] for multipath data transmission. The fitness function is formed based on energy, inter-cluster and intra-cluster delay, link quality, hop count and lifetime.

Delay is computed for path selection as the sum of inter-cluster delay ( delay $\left(P_{d \text { (inter) }}\right)$ and intracluster delay (delay $\left(P_{d(\text { intra })}\right)$. It is given by

$$
\begin{aligned}
\text { Path delay }\left(P_{d}\right) & =\operatorname{delay}\left(P_{d(\text { inter })}\right. \\
& \left.+\operatorname{delay}\left(P_{d(\text { intra })}\right)\right)
\end{aligned}
$$

Both intra-cluster delay and inter-cluster delay are computed based on the time for transmitted packets to reach destination in intra-cluster and inter-clusters respectively. The hop count is computed as the number of adjacent forwarding nodes in transmission. For a path with transmission range $\mathrm{W}$ and density of node deployment $(\rho)$, hop count can be estimated as 


$$
\text { Hop }=\left\lceil\frac{\text { Distance to destination }}{\frac{W}{2} \cos \left(\frac{1}{2} \arcsin \frac{4}{\rho W^{2}}\right)}\right\rceil-1
$$

Here $\left[\frac{D}{\frac{W}{2} \cos \left(\frac{1}{2} \arcsin \frac{4}{\rho W^{2}}\right)}\right]$ is the expected number of regions.

The fitness function for path selection $\left(F_{p}\right)$ is formulated similar to Eq. (10) of $\mathrm{CH}$ selection and it is given by

$$
\begin{gathered}
F_{p}=w\left(P_{d}\right) \times f\left(P_{d}\right)+w(e) \times f(e) \\
+w(\mathbb{E}[L]) \times f(\mathbb{E}[L])+w\left(L_{q}\right) \times f\left(L_{q}\right) \\
+w(H o p) \times f(H o p)
\end{gathered}
$$

Here $f\left(P_{d}\right)$ and $f(H o p)$ are the functions of path delay and hop count while $w\left(P_{d}\right)$ and $w(H o p)$ are weight functions of path delay and hop count, respectively.

The roosters with highest fitness values are selected as the leader of the group with high priority in accessing the food source. For ease, this case is performed via the location that the roosters with improved objective values can search for food in a broad range of locations than that of the roosters with worst fitness values. This rooster movement and location update can be given as

$$
X_{i, j}^{T+1}=X_{i, j}^{T} \times\left(1+\operatorname{rand}\left(0, \sigma^{2}\right)\right)
$$

Where $\operatorname{rand}\left(0, \sigma^{2}\right)$ is a Gaussian distribution with mean 0 and standard deviation $\sigma^{2}, X_{i, j}^{T}$ is a location of current best chicken for food source and $X_{i, j}^{T+1}$ is the location of next best chicken.

Since the hens have to take care of the young chicks, they can search food through their groupmate roosters and can arbitrarily take the highquality food found by other chickens. The new leading hens include the benefit in competing for food than the new passive ones. These phenomena of the hen formulate the movement and location update which are given as:

$$
\begin{gathered}
X_{i, j}^{T+1}=X_{i, j}^{T}+S_{1} \times \operatorname{rand} \times\left(X_{r 1, j}^{T}-X_{i, j}^{T}\right) \\
+S_{2} \times \text { rand } \times\left(X_{r 2, j}^{T}-X_{i, j}^{T}\right)
\end{gathered}
$$

Where Rand is a uniform random number over $[0,1]$, $S_{1}, S_{2}$ are the coefficient of social factors in search space; $r 1 \in[1, \ldots, N]$ is an index of the rooster and $r 2 \in[1, \ldots, N]$ is an index of the chicken (rooster or hen) randomly chosen from the swarm with $r 1 \neq r 2$. $S_{2}<1<S_{1}$ and i, j are hen's group mate. $S_{1}$ and $S_{2}$ are computed as $S_{1}=\exp \left(\left(f_{i}-f_{r 1}\right) /\left(a b s\left(f_{i}\right)+\right.\right.$ $\varepsilon))$ and $S_{2}=\exp \left(f_{r 2}-f_{i}\right)$. Here $f_{i}, f_{r 1}$ and $f_{r 2}$ are the fitness of i-th hen, rooster with index $r 1$ and rooster/hen with index $r 2$ while $a b s\left(f_{i}\right)$ is the absolute value of fitness and $\varepsilon$ is the smallest constant to avoid zero-division error.

The chicks move around their mother to forage for food. This is given as the movement and location update equation.

$$
X_{i, j}^{T+1}=X_{i, j}^{T}+f l \times\left(X_{m, j}^{T}-X_{i, j}^{T}\right)
$$

Where $X_{m, j}^{T}$ is the location of the $\mathrm{j}$-th chick's mother (index $\mathrm{m}$ belongs to $[1, \mathrm{~N}]$ ), $f l$ is a parameter, that the chick pursue its mother to forage for food. Assuming the individual differences, the value of $f l$ of every chick would be randomly chosen between 0 and 2. Depends on these values and the residual energy, a new route is determined over the network from source to destination. The overall route selection process is summarized in algorithm 2 .

\section{Algorithm 2. CSO based route selection}

Form paths for routing

Initialize a population of $\mathrm{N}$ chickens and define the related parameters;

Assign the paths as chickens

Evaluate the N chickens' fitness values using Eq. (19), iterations $\mathrm{m}=0$;

While ( $\mathrm{m}<$ Max_Generation)

If ( $\mathrm{m} \% \mathrm{G}==0) \quad / / / \mathrm{m}$ is a fraction of $\mathrm{G}$ steps of chick movement

Rank the chickens' fitness values and launch a hierarchal swarm order;

Classify the swarm into many groups of paths

Fix the relationship amongst the chicks and mother hens in a group;

Compare the paths i, i+1;

Rank the paths to transmit packets

Until termination conditions achieved

End if

For $\mathrm{i}=1: \mathrm{N}$

If $\mathrm{i}==$ rooster, then update its location via (20)

End if

If $i==$ hen, then update its location via (21)

End if

If $i==$ chick, then update its location via (22)

End if

Evaluate the new solution;

If the new solution is superior than its previous one, replace previous solution;

Obtain the multiple shortest optimal routes by destination

Assign each group for multi-path transmission

End for

End while 
Table 2. Simulation parameters

\begin{tabular}{|l|l|}
\hline Simulator & NS-2.34 \\
\hline No. of Nodes & 100 \\
\hline Area Size & 1000 X $1000 \mathrm{~m}$ \\
\hline Channel type & Wireless Channel \\
\hline Propagation model & Two Ray Ground \\
\hline Link Layer & LL \\
\hline Antenna model & Omni Antenna \\
\hline Traffic type & CBR \\
\hline Mobility model & Random Way point \\
\hline MAC & 802.11 \\
\hline Initial energy & 100 Joules \\
\hline Radio Range & $250 \mathrm{~m}$ \\
\hline Simulation Time & 300 seconds \\
\hline Number of packets & 1000 \\
\hline Packet rate & 8 packets/sec \\
\hline Data payload & 512 bytes/packet \\
\hline
\end{tabular}

CSO minimizes the energy drain rate, inter and intra cluster delay, and hop count nodes to build best shortest path route. In the proposed WSN based IoT network, all nodes are worked cooperatively and efficiently by sharing multiple information based on inter and intra cluster delay, link quality of the node and partial routes. Hence the FFA based CSO utilizes the best $\mathrm{CH}$ for the multipath routing through multiple objectives.

\section{Experimental results}

The proposed FFA+CSO based routing model is simulated in NS-2 simulator. The existing models namely Fractional Artificial Bee Colony-Enhanced Ant Colony Optimization (FABC+EACO) [16], Fractional Gravitational Search AlgorithmFractional Grey Wolf Optimization (FGSA+FGWO) [17], and FA+CSO are compared in terms of end to end delay, throughput, energy consumption, hopcount, PSNR and network lifetime parameters. The simulation settings are given in Table 1 .

\subsection{Performance metrics}

(i) End-to-end delay is estimated as the average time taken by the packets to transmit from source to the destination nodes across the network and it includes the inter-cluster and intra-cluster delay, buffer delay, queuing delay, etc. High delay results in the packet loss and subsequent energy wastage for the retransmissions.

(ii) Throughput is the rate of successful data packets transmission in a network. It is estimated in bits per second (bit/s or bps). It is also specified by units of information processed over a given time slot. It can be computed by

Throughput $=$

$\frac{\sum \text { Number of packets recieved by destination }}{\text { Simulation time }} \times \frac{8}{1000}$

(iii) Energy consumption refers to the average energy necessary for transmitting, receiving or forwarding operations of a packet to a node in the network during a period of time. It is measured in Joules per second.

(iv) Network lifetime is the total lifespan of a network calculated by the time to first dead node or a group of nodes in the network.

(v) PSNR value should be high for the proposed method. It is defined to determine the quality of received data packets and is estimated as the ratio of maximum power of received packet to the noise associated with it. It is computed by

$$
\operatorname{PSNR}(\mathrm{dB})=20 \log _{10} \frac{2^{\mathrm{s}}-1}{\sqrt{\mathrm{MSE}}}
$$

Here MSE is the mean square error given by $\operatorname{MSE}=\frac{1}{\mathrm{~N}_{1} \times \mathrm{N}_{2}} \sum_{\mathrm{i}}^{\mathrm{N}_{1}} \sum_{\mathrm{i}}^{\mathrm{N}_{2}}[\mathrm{X}(\mathrm{i}, \mathrm{j})-\widehat{\mathrm{X}}(\mathrm{i}, \mathrm{j})]^{2}$ with $X(i, j)$ denoting the data objects.

(vi) Hop count: In networking, a hop count is the total sum of transitional devices such as routers or nodes via which information must pass from the source to destination, rather than flowing straight over a single wire. Along the information path, every node forms a hop, with data moving from one node to another node.

\subsection{Results and discussion}

Fig. 1 illustrates the comparison of (a) end to end delay, (b) throughput, (c) energy consumption, (d) network lifetime, (e) PSNR and (f) hop count results between the FABC+EACO, FGSA+FGWO, $\mathrm{FA}+\mathrm{CSO}$ and the proposed $\mathrm{FFA}+\mathrm{CSO}$ routing models. Fig. 1(a) shows that the proposed FFA+CSO model has less delay than the other models because of the selection of optimal $\mathrm{CH}$ and routes that reduces the overall time for data transmission and ensures energy efficient delay-less transmission. For instance, while the number of nodes is 100 , the delay of FFA+CSO is $0.95 \mathrm{~ms}$ 


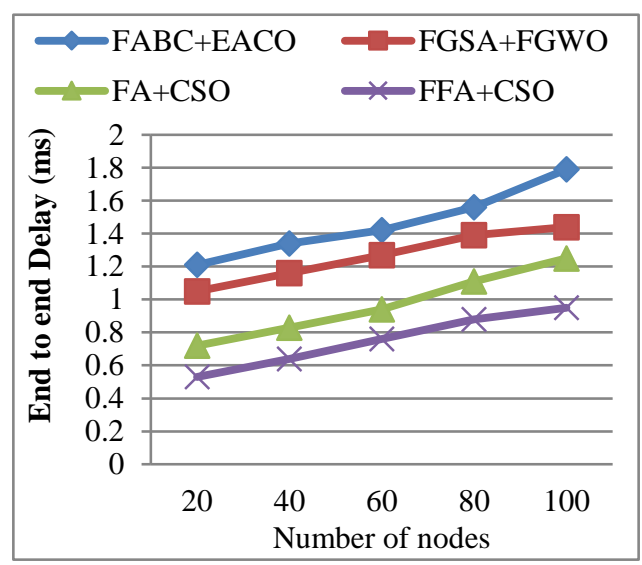

(a)

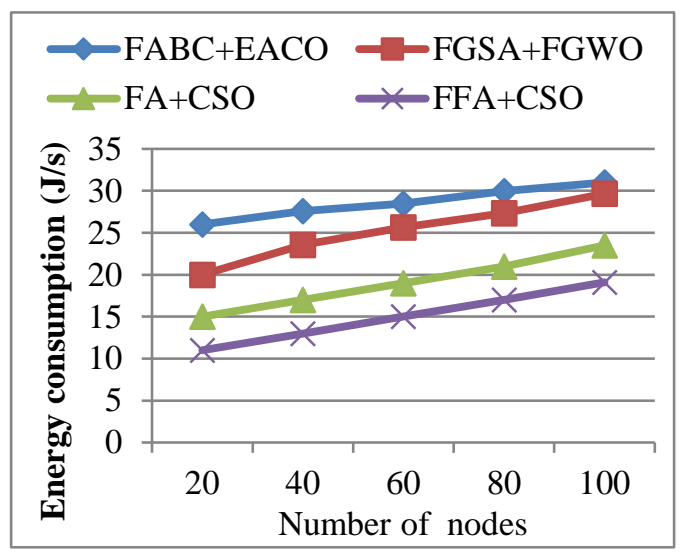

(c)

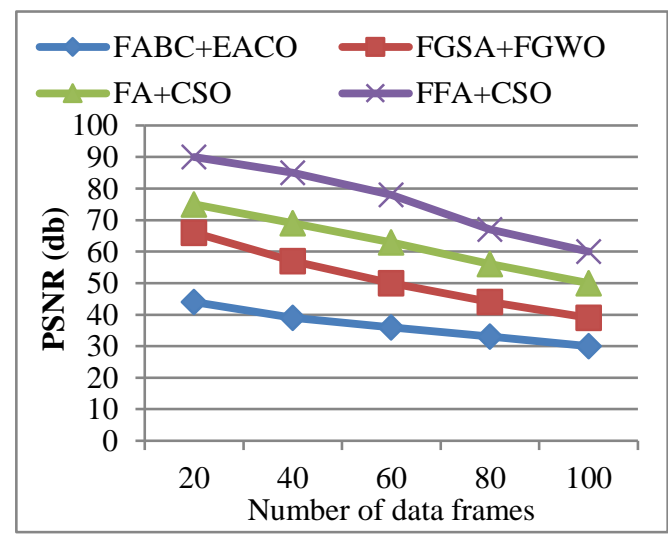

(e)

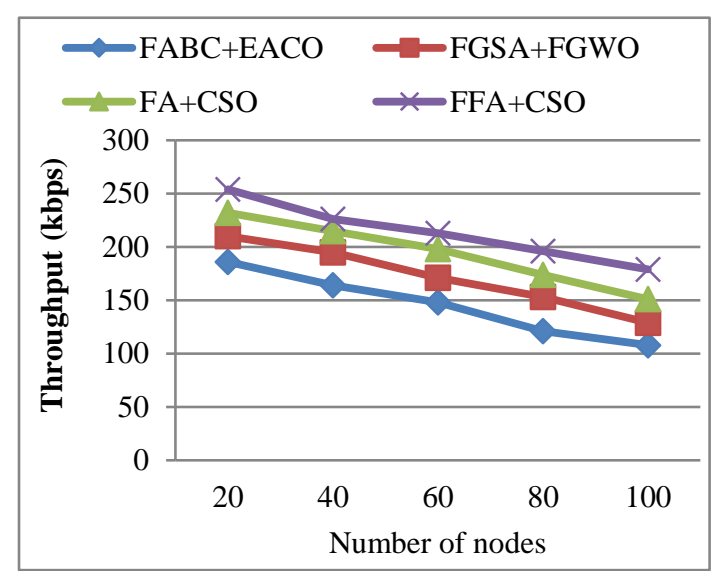

(b)

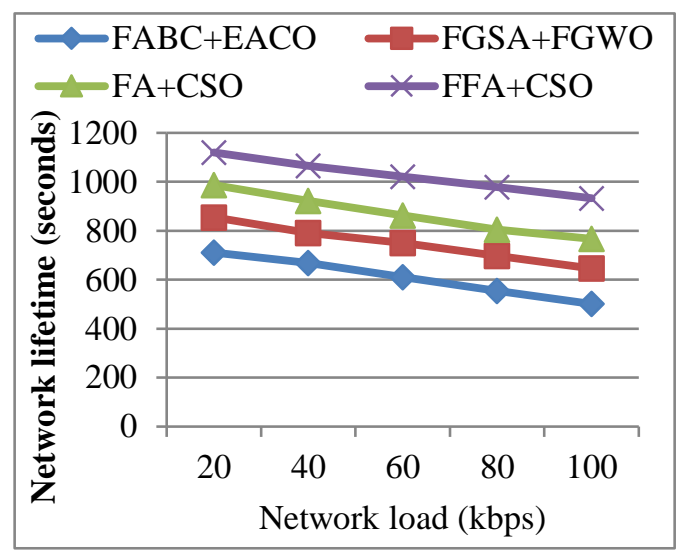

(d)

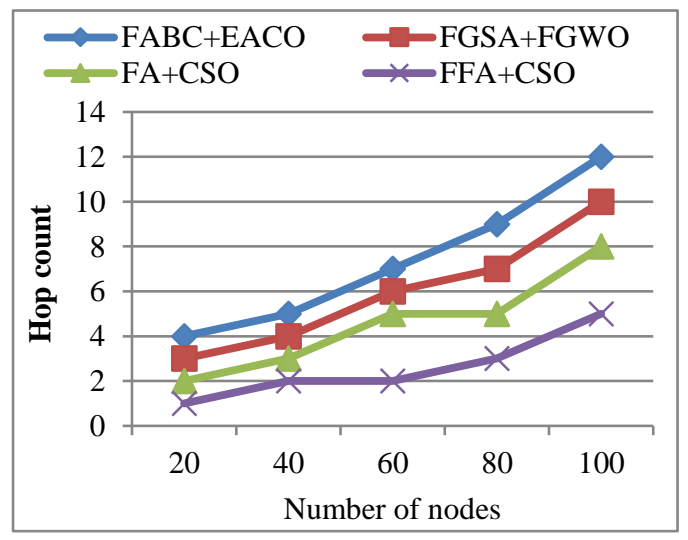

(f)

Figure. 1 Performance comparison: (a) end-to-end delay, (b) throughput, (c) energy consumption, (d) network lifetime, (e) PSNR, and (f) hop count

which is around $24 \%$ less than the second best FA+CSO model while $34 \%$ and $46 \%$ less than FGSA+FGWO and FABC+EACO models respectively.

Fig. 1 (b) shows that the proposed FFA+CSO model has high throughput than the other models.
For instance, while the number of nodes is 100 , the throughput of FFA+CSO is $179 \mathrm{kbps}$ which is around $28 \%, 38 \%$ and $65 \%$ greater than the $\mathrm{FA}+\mathrm{CSO}, \quad \mathrm{FGSA}+\mathrm{FGWO}$ and $\mathrm{FABC}+\mathrm{EACO}$ models respectively. This is because of the reliable multi-path transmission of $\mathrm{FFA}+\mathrm{CSO}$ in ensuring 
energy-efficient data transmission with reduced need for packet retransmission due to packet loss. From Fig. 1 (c), it is observed that the proposed FFA+CSO have low energy consumption than the other models. When the number of nodes is 100 , the energy consumption of FFA+CSO is $19.1 \mathrm{~J} / \mathrm{s}$ which is $18.7 \%, 35 \%$ and $38 \%$ lesser than the $\mathrm{FA}+\mathrm{CSO}$, FGSA+FGWO and FABC+EACO models respectively. The better energy conservation is due to the uniform energy drain rate through balanced energy consumption of FFA+CSO model.

Fig. 1 (d) shows that the proposed FFA+CSO model has high network lifetime due to effective resolving of the energy-hole problem and the nonuniform energy dissipation problem. When compared at $100 \mathrm{kbps}$ of network load, the network lifetime of FFA+CSO is $933 \mathrm{~s}$ which is $21.6 \%$, $44.4 \%$ and $86 \%$ higher than the $\mathrm{FA}+\mathrm{CSO}$, FGSA+FGWO and FABC+EACO models respectively.

Fig. 1 (e) illustrate that the proposed FFA+CSO model has high PSNR than other models due to the minimum loss of packets during transmission. When compared at 100 data frames, the PSNR of FFA+CSO is $60 \mathrm{~dB}$ which is $20 \%$ higher than the second best FA+CSO model due to its superior routing behaviour.

Similarly, from Fig. 1 (f), it can be observed that the proposed FFA+CSO model has less hop count than other models due to energy-efficient and optimal path selection. When compared at 100 nodes, the FFA+CSO has 5 hops which is $37.5 \%$ less than the second best FA+CSO model due to its superior routing behaviour. Similarly, it also outperforms the FABC+EACO and the FGSA+FGWO models. The competence of the $\mathrm{FFA}+\mathrm{CSO}$ is attributed to the fractional firefly based $\mathrm{CH}$ selection which improves the exploration ability of the FA algorithm and hence increases the efficacy of the routing models. These results prove the significance of the proposed FFA+CSO routing model for efficient data transmission in WSN based IoT applications.

\section{Conclusion and future work}

This paper presented the development of energy proficient routing protocol using $\mathrm{FFA}+\mathrm{CSO}$ for multipath data transmission in WSN based IoT networks. The proposed FFA+CSO model utilized FFA for optimal CH selection and CSO for optimal route selection. This model averted the problems of energy-hole problem and non-uniform energy dissipation issue by balancing the energy and load so that single path does not suffer from early expiration of nodes. The simulation results performed in NS-2 proved that the proposed FFA+CSO routing model outperforms the other existing routing models significantly with $24 \%$ less delay, $28 \%$ greater throughput, $18.7 \%$ lesser energy consumption, $21.6 \%$ longer lifetime, $20 \%$ higher PSNR and $37.5 \%$ less number of hops for evaluation with 100 nodes. This proves that the FFA+CSO are more suitable for smart applications of WSN based IoT networks.

In future, the multi-objective optimization problem can be extended to include more parameters without increasing the cost and complexity using novel adaptive and advanced optimization principles. Another direction for future research is the extending the proposed FFA+CSO model to achieve fault tolerance in WSN based IoT without considerably affecting data routing and endto-end delay.

\section{References}

[1] J. Gubbi, R. Buyya, S. Marusic, and M. Palaniswami, "Internet of Things (IoT): A vision, architectural elements, and future directions", Future Generation Computer Systems, Vol. 29, No. 7, pp. 1645-1660, 2013.

[2] D. Bandyopadhyay and J. Sen, "Internet of things: Applications and challenges in technology and standardization", Wireless Personal Communications, Vol. 58, No. 1, pp. 49-69, 2011.

[3] M. T. Lazarescu, "Design of a WSN platform for long-term environmental monitoring for IoT applications", IEEE Journal on Emerging and Selected Topics in Circuits and Systems, Vol. 3, No. 1, pp. 45-54, 2013.

[4] M. Azharuddin, P. Kuila, and P. K. Jana, "Energy efficient fault tolerant clustering and routing algorithms for wireless sensor networks", Computers and Electrical Engineering, Vol. 41, No. 2015, pp. 177-190, 2015.

[5] Z. Sheng, C. Mahapatra, C. Zhu, and V. C. Leung, "Recent advances in industrial wireless sensor networks toward efficient management in IoT", IEEE Access, Vol. 3, pp. 622-637, 2015.

[6] N. A. Pantazis, S. A. Nikolidakis, and D. D. Vergados, "Energy-efficient routing protocols in wireless sensor networks: A survey", IEEE Communications Surveys and Tutorials, Vol. 15, No. 2, pp. 551-591, 2012.

[7] J. Rahman, M. A. M. Hasan, and M. K. B. Islam, "Comparative analysis the performance 
of AODV, DSDV and DSR routing protocols in wireless sensor network", In: Proc. of the 7th International Conference on Electrical and Computer Engineering, pp. 283-286, 2012.

[8] T. Rault, A. Bouabdallah, and Y. Challal, "Energy efficiency in wireless sensor networks: A top-down survey", Computer Networks, Vol. 67, No. 1, pp. 104-122, 2014.

[9] K. Machado, D. Rosário, E. Cerqueira, A. Loureiro, A. Neto, and J. de Souza, "A routing protocol based on energy and link quality for internet of things applications", Sensors, Vol. 13, No. 2, pp. 1942-1964, 2013.

[10] J. Shen, A. Wang, C. Wang, P. C. Hung, and C. F. Lai, "An efficient centroid-based routing protocol for energy management in WSNassisted IoT", IEEE Access, Vol. 5, No. 1, pp. 18469-18479, 2017.

[11] T. D. Nguyen, J. Y. Khan, and D. T. Ngo, "An effective energy-harvesting-aware routing algorithm for WSN-based IoT applications", In: Proc. of IEEE International Conference on Communications (ICC), pp. 1-6, 2017.

[12] T. D. Nguyen, J. Y. Khan, and D. T. Ngo, "A distributed energy-harvesting-aware routing algorithm for heterogeneous IoT networks", IEEE Transactions on Green Communications and Networking, Vol. 2, No. 4, pp. 1115-1127, 2018.

[13] B. Singh and D. K. Lobiyal, "Energy-aware cluster head selection using particle swarm optimization and analysis of packet retransmissions in WSN", Procedia Technology, Vol. 4, No. 1, pp. 171-176, 2012.

[14] K. Rana and M. Zaveri, "Synthesized cluster head selection and routing for two tier wireless sensor network", Journal of Computer Networks and Communications, Vol. 2013, No. 1, pp. 1-11, 2013.

[15] P. S. Rao, P. K. Jana, and H. Banka, "A particle swarm optimization based energy efficient cluster head selection algorithm for wireless sensor networks", Wireless Networks, Vol. 23, No. 7, pp. 2005-2020, 2017.

[16] R. Kumar, D. Kumar, and D. Kumar, "Exponential Ant Colony Optimization and Fractional Artificial Bee Colony to Multi-Path Data Transmission in Wireless Sensor Networks", IET Communications, Vol. 11, No. 4, pp. 522-530 2017.

[17] A. V. Dhumane and R. S. Prasad, "Fractional Gravitational Grey Wolf Optimization to MultiPath Data Transmission in IoT", Wireless Personal Communications, Vol. 102, No. 1, pp. 411-436, 2018.
[18] J. Luo, D. Wu, C. Pan, and J. Zha, "Optimal energy strategy for node selection and data relay in WSN-based IoT", Mobile Networks and Applications, Vol. 20, No. 2, pp. 169-180, 2015.

[19] V. K. Verma, S. Singh, and N. P. Pathak, "Analysis of scalability for AODV routing protocol in wireless sensor networks", Optik, Vol. 125, No. 2, pp. 748-750, 2014.

[20] X. S. Yang, "Multi-objective firefly algorithm for continuous optimization", Engineering with Computers, Vol. 29, No. 2, pp. 175-184, 2013.

[21] J. A. Tenreiro Machado, M. F. Silva, R. S. Barbosa, I. S. Jesus, C. M. Reis, M. G. Marcos, and A. F. Galhano, "Some applications of fractional calculus in engineering", Mathematical Problems in Engineering, Vol. 2010, No. 1, pp. 1-34, 2010.

[22] X. Meng, Y. Liu, X. Gao, and H. Zhang, "A new bio-inspired algorithm: chicken swarm optimization", In: Proc. of International Conference in Swarm Intelligence, pp. 86-94, 2014. 